\title{
Gastric Metastasis and Transformation of Primary Lung Adenocarcinoma to Small Cell Cancer After Acquired Resistance to EGFR-Tkls: A Case Report
}

Jing Liu

The Second Affiliated Hospital of Chongqing Medical University https://orcid.org/0000-0001-71009078

Lei Xia

The Second Affiliated Hospital of Chongqing Medical University

Yuan Peng

The Second Affiliated Hospital of Chongqing Medical University

YuSheng Huang

The Second Affiliated Hospital of Chongqing Medical University

ZhenZhou Yang ( $\square$ yangzhenzhou@sohu.com )

The Second Affiliated Hospital of Chongqing Medical University

\section{Case Report}

Keywords: small cell lung cancer, transformation, neoadjuvant/adjuvant EGFR-TKIs treatment, gastric metastasis, case report

Posted Date: June 11th, 2021

DOI: https://doi.org/10.21203/rs.3.rs-606938/v1

License: (9) This work is licensed under a Creative Commons Attribution 4.0 International License. Read Full License 


\section{Abstract}

Background: Transformation to small cell lung cancer (SCLC) is one of the mechanisms of resistance to epidermal growth factor receptor tyrosine kinase inhibitors (EGFR-TKIs). However, no standard treatment is available after the transformation. In addition, gastric metastasis of primary lung cancer is rarely observed, thus, little is known about its metastasis characteristics.

Case presentation: A 58-year-old male patient was treated with osimertinib (80 mg/day) as the 2 st line treatment due to recurrence and metastasis after surgical resection first-line gefitinib for EGFR exon 19 mutation pulmonary adenocarcinoma. 1 year and 3 months after osimertinib initiation, all the lesions are increased. He complained stomachache, and a diagnostic gastroscopy biopsy confirmed small cell lung cancer in the gastric body, indicating osimertinib-induced phenotypic transformation. He was treated with etoposide and los platinum chemotherapy and the maintenance therapy with osimertinib. Finally, the patient achieved a partial response after four cycles.

Conclusions: Timely second biopsy should be valued in the diagnosis of phenotypic transformation. After transformation, a chemotherapeutic treatment with etoposide and platinum and maintenance therapy with osimertinib inhibited the progress of the disease.

\section{Background}

The use of EGFR-TKls has been approved as a treatment against advanced non-small-cell lung carcinoma (NSCLC) with EGFR-activating mutations. However, almost all patients treated with EGFR-TKIs eventually develop resistance to these agents. The T790M mutation plays an important role in the acquired resistance to the treatment with first- or second-generation EGFR-TKIs. If an NSCLC patient acquired a T790M mutation, the third-generation EGFR-TKI osimertinib is firstly recommended [1]. This treatment induced a remarkable response compared with standard cytotoxic chemotherapy in patients with EGFR-positive NSCLC [2,3]. However, despite this favourable response, these patients experience disease progression after an average time of approximately 12 months [4]. Several resistance mechanisms have been identified, including EGFR C797S mutation, EGFR MET or HER2 amplification, phosphoinositide 3- kinase pathway activation, and rare transformation from lung adenocarcinoma (LUAD) to SCLC $[5,6,7,8]$. Transformation to SCLC is considered as a mechanism of resistance to EGFR-TKIs treatment in approximately $5 \%$ of patients [9].

Patients with stage IIIA-N2 NSCLC harbor a considerable heterogeneity with variable ipsilateral mediastinal lymph node involvement (Robinson classification IIIA1 to IIIA4) [10]. The treatment includes the following options: surgery followed by adjuvant chemotherapy, neoadjuvant therapy followed by surgical resection, or definitive chemoradiation. Downstaging of N2 disease with neoadjuvant chemotherapy is associated with an approximately $70 \%$ resectability rate [11]. However, EGFR-TKIs significantly prolong the progression-free survival of patients with advanced NSCLC positive for EGFR mutation compared with chemotherapy as first-line treatment $[12,13,14,15,16]$. The potential 
efficacy of neoadjuvant EGFR-TKIs therapy in patients with resectable NSCLC is described in case reports and small-scale nonrandomized clinical trials $[17,18,19,20]$. Zhong et al recently described the results of the randomized phase II EMERGING-CTONG 1103 trial (ClinicalTrials.gov identifier: NCT01407822) that explored the safety and efficacy of erlotinib compared with gemcitabine plus cisplatin (GC chemotherapy) as a neoadjuvant treatment in patients with stage IIIA-N2 NSCLC with EGFR mutations in the exon 19 or 21 [21, 22]. Their results showed that preoperative neoadjuvant targeted therapy could improve the complete resection rate and removal of micrometastases in the blood in these patients with stage II - IIIA (N1-N2) NSCLC.

Gastric metastasis from lung cancer is a relatively rare development. Indeed, its frequency ranges from $0.19 \%$ to $5.1 \%[23,24]$. The most common site of extra pulmonary spread includes liver $(35 \%)$, bones $(25 \%)$, adrenals $(22 \%)$, kidneys $(10-15 \%)$ and heart pericardium (20\%) [25]. However, the reported incidence of gastric metastasis from LUAD at post-mortem is relatively high, ranging from $4.7 \%$ to $14 \%$ $[26,27]$. Hence, this report describes a case of osimertinib resistance due to SCLC transformation and the gastric metastasis, the transformation from primary LUAD to SCLC, their features, prognosis and optimal treatment strategy after gastric metastasis were discussed.

\section{Case Presentation And Results}

A 58-year-old male Chinese patient, with no smoking history (only passive smoking), was admitted to the Third military medical university affiliated first hospital (Chongqing, Chinese) because of cough and expectoration (Fig. 1). The scan of the chest and abdomen by computed tomography (CT) revealed the presence of a primary tumor (with the longest diameter measuring $52 \mathrm{~mm}$ ) in the right lower lobe (Fig. 2a) and subcarinal lymph node metastasis (Fig. 2b). The lung biopsy revealed the presence of adenocarcinoma cells (Fig. 2c). The clinical tumor stage was evaluated as T3N2M0 (stage IIIB). The potential presence of EGFR mutations was evaluated using a pre-operation biopsy specimen and the results revealed an exon 19 deletion in the EGFR gene. Hence, the patient was subjected to a neoadjuvant therapy with the first-generation EGFR-TKI gefitinib (0.25 g). After 2 months (Jul 2017), the CT scanning of the chest performed at the Third military medical university affiliated third hospital (Chongqing, China) revealed the reduction of the right lower lobe mass (with the longest diameter measuring $31 \mathrm{~mm}$ ) (Fig. 3a). The surgery "right lung middle lower lobe resection and mediastinum and hilum lymph node cleaning technique" was performed (Fig. 3b) (Jul 2017). The postoperative pathologic staging was pT2aNOM0 (stage IB). Subsequently, the patient was subjected to four cycles of chemotherapy composed of pemetrexed $800 \mathrm{mg}$ ( $500 \mathrm{mg} / \mathrm{m} 2$ intravenously injected at day 1 , once every 3 weeks) combined with nida's platinum $60 \mathrm{mg}$ ( $80 \mathrm{mg} / \mathrm{m} 2$ intravenously injected at day 1-2, once every 3 weeks).

After six months (Apr 2018), the patient complained of headaches, and brain magnetic resonance imaging (MRI) revealed the progression of the disease, with three lesions in the left parietal lobe, temporal lobe, and right frontal lobe. The patient continued to be treated with the first-generation EGFR-TKI gefitinib $(0.25 \mathrm{~g})$, achieving a partial response after 2 Mon and all lesions were treated with Gamma Knife radiosurgery at a dose of $16 \mathrm{~Gy}$ (measuring curve of $50 \%$ ). 
At 5 months after gefitinib (0.25 g) treatment (Sep 2018), the patient showed disease progression with a metastatic lesion in the right kidney. Thus, the therapy was subsequently replaced with standard pemetrexed $800 \mathrm{mg}$ (500 mg/m2 intravenously injected at day 1, once every 3 weeks) combined with nida's platinum $60 \mathrm{mg}(80 \mathrm{mg} / \mathrm{m} 2$ intravenously injected at day 1-2, once every 3 weeks) (PP regime). The patients experienced loss of appetite and general weakness after three cycles and the disease further progressed involving the left kidney with another metastasis (Nov 2018). Therefore, the treatment was adjusted according to a personalized therapy, using bevacizumab $500 \mathrm{mg}(7.5 \mathrm{mg} / \mathrm{kg}$ intravenously injected at day 1 , once every 3 weeks) combined with pemetrexed $800 \mathrm{mg}(500 \mathrm{mg} / \mathrm{m} 2$ intravenously injected at day 1 , once every 3 weeks). The patient achieved a stable disease after one cycle. However, the patient was not satisfied with the curative effect and asked to change the therapy. Thus, the treatment was adjusted one more time to enhance the curative effect, and bevacizumab $500 \mathrm{mg}(7.5 \mathrm{mg} / \mathrm{kg}$ intravenously injected at day 1 , once every 3 weeks) combined with paclitaxel liposome $240 \mathrm{mg}$ (135 $\mathrm{mg} / \mathrm{m} 2$ intravenously injected at day 1 , once every 3 weeks) was used. Blood samples were collected and the molecular testing of EGFR by amplification refractory mutation system-polymerase chain reaction detected an EGFR T790M mutation. Therefore, the patient was treated with osimertinib, and the progression of the disease was observed after 1 year and 3 months (Apr 2020). The CT scan of the chest and abdomen revealed a secondary tumor (with the longest diameter measuring $12 \mathrm{~mm}$ ) in the right upper lobe and a steady nodule in the right kidney. Brain MRI revealed that the brain lesions have not change. Blood samples were collected again and subjected to next-generation sequencing, which revealed the presence of an EGFR T790M mutation and the original exon 19 deletion simultaneously. Subsequently, the patient was treated with hypofractionated radiotherapy in the right upper lobe (50 Gy/5 $\mathrm{Gy} / 10 \mathrm{f}$ ) and continued the treatment with osimertinib. Unfortunately, the CT performed after 2 months (Jun 2020) revealed a disease progression due to the increase of the nodules in the right upper lobe posterior segment and right kidney compared with the previous situation. MRI revealed the enlargement of the lesions in the left after ventricle angle compared with the previous situation. Thus, the patient subsequently was treated with hypofractionated radiotherapy in the left after ventricle angle (44 Gy/4 $\mathrm{Gy} / 11 \mathrm{f}, 3 \mathrm{~Gy} / 1 \mathrm{f}$ ) and in the right upper lobe posterior segment (50 Gy/5 Gy/10 f). After radiotherapy, he developed a stomachache, and the gastroscopy with biopsy showed a small cell carcinoma in the gastric body derived from the lung (Fig. 4a-b). Blood samples again were collected and the NGS detected an exon 19 deletion in the EGFR and T790M mutation. The pathological examination after tissue staining showed that the metastatic tumor cells were localized in the submucosa of the stomach (Fig. 4c). Therefore, the patient was subjected to a standard treatment with etoposide $130 \mathrm{mg}$ (100 mg/m2 intravenously injected at day 1-3, once every 3 weeks) and los platinum $45 \mathrm{mg}$ (30 mg/m2 intravenously injected at day 1 , once every 3 weeks) (EP) chemotherapy and the maintenance therapy with osimertinib. Finally, the patient achieved a partial response after four cycles. The lesions in the left kidney and right kidney before chemotherapy are shown in fig. 6a-b. while the left and right kidney lesions achieved a partial response after a four-cycle treatment (Fig. $6 \mathrm{~d}-\mathrm{e}$ ). The gastroscopy revealed that the gastric lesion was disappeared (Fig. 6c), but the biopsy of the mucosa still showed signs of a malignant tumor (Fig. 6f). The brain and lung lesions achieved a stable disease state. In addition, the patient showed a disease progression a enlarged lesion lesion in the stomach after termination of chemotherapy. The surgery 
"partial gastrectomy" was performed. The potential presence of an exon 19 deletion in the EGFR and TP53 mutations was evaluated using gastric postoperative specimens and blood samples.

\section{Discussion And Conclusions}

Gastric metastasis from primary lung carcinoma is a rare phenomenon. The most common type of cells in the gastric metastasis are squamous cell carcinoma cells [28]. However, gastric metastasis from primary LUAD is an unusual phenomenon. The cases of gastric metastasis from primary LUAD cancer previously reported in the literature are summarized in Table 1. According to these previous cases, only four had a single metastasis in the stomach. Our study showed that the metastatic tumor was found above the body of the stomach. The esophagogastroduodenoscopy showed that most cases of metastasis present as submucosal tumors with mucosal folds up and modest ulcerations towards the top are often referred to as volcano-like ulcers. In general, tumors with these characteristics are regarded as hematogenous or lymphatic metastatic lesions, but this aspect was not clarified in the previous reports. The occurrence of gastric metastasis from LUAD will greatly increase, although the cause is not clear. Two hypotheses have been postulated. One is that the sputum containing cancer cells is swallowed into the digestive tract, especially in smokers who are more susceptible to gastric mucosal damage [44]. Thus, Helicobacter pylori infection and/or ulcers may be associated with gastric metastasis. The other is that some cytokines may affect the organ specificity of blood metastasis [45]. Our hypothesis is that our case was a hematogenous metastasis with a severe vascular, but not lymphatic invasion, as histologically detected around the tumor. In addition, the transformation to SCLC in patients with EGFRsensitive mutation is not rare. A study analyzed 103 patients with T790M positive mutation after the first generation of TKI resistance who were treated with osimertinib, and the results showed that the main mutations after osimertinib resistance were EGFR abnormality (25\%), MET amplification (16\%), TP53 mutation (8\%), KRAS mutation (4\%), RET fusion (4\%), HER2 amplification (4\%), and RB1 amplification (6.25\%) [46]. RB1 mutation indicates the transformation from LUAD to SCLC. In our case, the gastroscopy with biopsy indicated tumor cell infiltration, and immunohistochemistry confirmed that the small-cell cancer was originated from the lung. The immunohistochemical staining of CD56, TTF-1, $\mathrm{CgA}$ and CEA was positive, while EMA, CD3 and CD68 were negative, confirming the diagnosis of gastric small-cell cancer from LUAD. However, the problem was that the patient underwent pathological changes after or before gastric metastasis from the lung. Our hypothesis was that the gastric metastasis developed after the transformation. Firstly, the immunohistochemical staining showed that the tumor cells near the submucosa were significantly bigger than those near the muscle layer (Fig. 5a).

Furthermore, $\mathrm{CgA}$ expression was more remarkable in the submucosa and less in the muscular layer (Fig. $5 b)$. On the contrary, CK7 expression was more remarkable near the muscular layer and less in the submucosa (Fig. 5c). This suggested that the small cell transformation already occurred before the invasion. Secondly, the lesions in the kidney continued to increase after osimertinib treatment alone. The lesions in the kidney inversely resulted in a partial response using EP and the maintenance treatment with osimertinib (Fig. 6d-e). 
In addition, at the time of progression to osimertinib, due to phenotypic transformation, our patient received standard platinum-etoposide chemotherapy and the maintenance treatment with osimertinib, achieving clinical benefit. The identification of biomolecular mediators of treatment dependent SCLC transformation represents a fundamental goal to subsequently develop therapeutic interventions. Current evidence supports TP53 and RB1 mutations as potential predictors of phenotypic switch in EGFRmutated NSCLC [47]. Moreover, a rapid increase in the serum levels of neurone specifific enolase (NSE) together with a poor response to EGFR-TKIs usually indicates a transformation from adenocarcinoma to SCLC. In our case, TP53 mutations were detected by gastric tissue NGS analysis after osimertinib, suggesting that the patient possibly develop SCLC in the gastric. In conclusion, this report described a patient with EGFR mutant NSCLC small cell type transformation and gastric metastasis after osimertinib treatment, suggesting the necessity of another biopsy because of the non-classical metastasis in the stomach. Primary tumor another biopsy simultaneously could help us judge timely the happen sequence of transformation to SCLC and metastasis and initiation of appropriate treatment. Though less occurrence of lung cancer with gastric metastasis, attention should be paid when gastrointestinal symptoms occurred for patients with lung cancer. Timely detection of transformation to SCLC and initiation of appropriate treatment could improve the quality of life and prolong survival. A chemotherapeutic treatment combined with EGFR-TKIs should be the appropriate treatment in these patients with SCLC transformation.

\section{Abbreviations}

SCLC: small cell lung cancer; EGFR-TKIs: epidermal growth factor receptor tyrosine kinase inhibitors; LUAD: lung adenocarcinoma; NSCLC: non-small-cell lung carcinoma; CT: computed tomography; $\mathrm{MRI}=$ magnetic resonance imaging

\section{Declarations}

\section{Acknowledgment}

Not applicable.

\section{Author Contributions}

All authors made a significant contribution to the work reported, whether that is in the conception, study design, execution, acquisition of data, analysis and interpretation, or in all these areas; took part in drafting, revising or critically reviewing the article; gave final approval of the version to be published; have agreed on the journal to which the article has been submitted; and agree to be accountable for all aspects of the work.

\section{Funding}

Project supported by the National Natural Science Foundation of China (Grant No.81802984). 
Availability of data and materials

Main data generated or analysed during this study are included in this published article.

\section{Ethics approval and consent to participate}

Ethical approval is not required for this Case report. Written informed consent was obtained from the patient.

\section{Consent for Publication}

Written informed consent was obtained from the patient for publication of this paper and any accompanying images.

\section{Competing interests}

The authors declare that they have no conflicts of interest.

\section{Disclosure}

The authors have no competing interests to declare.

\section{References}

1. Mok TS. Wu YL, Thongprasert S, et al Gefitinib or carboplatin-paclitaxel in pulmonary adenocarcinoma. N Eng J Med. 2009;361(10):947-57.

2. Rossi A. LUX-Lung: determining the best EGFR inhibitor in NSCLC? Lancet Oncol. 2015;16(2):118-9., Di Maio M.

3. Engelman JA. Janne PA. Mechanisms of acquired resistance to epidermal growth factor receptor tyrosine kinase inhibitors in non-small cell lung cancer. Clin Cancer Res. 2008;14(10):2895-9.

4. Uchibori K. Inase N, Araki M, et al Brigatinib combined with anti-EGFR antibody overcomes osimertinib resistance in EGFR-mutated non-small-cell lung cancer. Nat Commun. 2017;8: 14768.

5. Le T. Sailors J, Oliver DH. Mayer M, Hoskin S. Gerber D. Histologic transformation of EGFR mutant lung adenocarcinoma without exposure to EGFR inhibition. Lung Cancer. 2017;105: 14-6.

6. Levin PA. Mayer M, Hoskin S. Sailors J, Oliver DH. Gerber DE. Histologic transformation from adenocarcinoma to squamous cell carcinoma as a mechanism of resistance to EGFR inhibition. $J$ Thorac Oncol. 2015;10 (9): e86-8.

7. Kogo M. Shimizu R, Uehara K, et al Transformation to large cell neuroendocrine carcinoma as acquired resistance mechanism of EGFR tyrosine kinase inhibitor. Lung Cancer. 2015;90: 364-8.

8. Oser MG. Niederst MJ, Sequist LV. Engelman JA. Transformation from non-small-cell lung cancer to small-cell lung cancer: molecular drivers and cells of origin. Lancet Oncol. 2015;16(4):e165-72. 
9. Zhong WZ. Chen KN, Chen C, et al Erlotinib Versus Gemcitabine Plus Cisplatin as Neoadjuvant Treatment ofStage IIIA-N2 EGFR-Mutant Non-Small-Cell Lung Cancer (EMERGING-CTONG 1103): ARandomized Phase II Study. J Clin Oncol. 2019;Jun13:JC01900075.

10. Robinson LA. Ruckdeschel JC, Wagner $\mathrm{H} J r$, Stevens $\mathrm{CW}$. Treatment of non-small cell lung cancerstage IIIA: ACCP evidence-based clinical practice guidelines (2nd edition). Chest. 2007;132:243S265S..

11. Rosell R. López-Cabrerizo MP, Astudillo J. Preoperative chemotherapy for stage IIIA non-small cell lung cancer. Curr Opin Oncol. 1997;9:149 - 55.

12. Fukuoka M. Wu YL, Thongprasert S, et al Biomarker analyses and final overall survival results from a phase III, randomized, open-label, first-line study of gefitinib versus carboplatin/paclitaxel in clinically selected patients with advanced non-small-cell lung cancer in Asia (IPASS). J Clin Oncol. 2011;29:2866-74.

13. Inoue A. Kobayashi $K$, Maemondo $M$, et al Updated overall survival results from a randomized phase III trial comparing gefitinib with carboplatin-paclitaxel for chemo-naïve non-small cell lung cancer with sensitive EGFR gene mutations (NEJ002). Ann Oncol. 2013;24:54 - 9.

14. Zhou C. Wu YL, Chen GY, et al Erlotinib versus chemotherapy as first-line treatment for patients with advanced EGFR mutation-positive non-small-cell lung cancer (OPTIMAL, CTONG-0802): A multicentre, open-label, randomised, phase 3 study. Lancet Oncol. 2011;12:735 - 42.

15. Wu YL. Zhou C, Liam CK, et al First-line erlotinib versus gemcitabine/cisplatin in patients with advanced EGFR mutation-positive non-small-cell lung cancer: Analyses from the phase III, randomized, open-label, ENSURE study. Ann Oncol. 2015;26:1883-9.

16. Zhong W. Yang X, Yan H, et al Phase II study of biomarker-guided neoadjuvant treatment strategy for IIIA-N2 non-small cell lung cancer based on epidermal growth factor receptor mutation status. $J$ Hematol Oncol. 2015;8:54.

17. Lara-Guerra H. Chung CT, Schwock J, et al Histopathological and immunohistochemical features associated with clinical response to neoadjuvant gefitinib therapy in early stage non-small cell lung cancer. Lung Cancer. 2012;76:235 - 41.

18. Lara-Guerra H. Waddell TK, Salvarrey MA, et al Phase II study of preoperative gefitinib in clinical stage I non-small-cell lung cancer. J Clin Oncol. 2009;27:6229-36.

19. Hishida T. Nagai K, Mitsudomi T, et al Salvage surgery for advanced non-small cell lung cancer after response to gefitinib. J Thorac Cardiovasc Surg. 2010;140:e69-71.

20. Schaake EE. Kappers I, Codrington HE, et al Tumor response and toxicity of neoadjuvant erlotinib in patients with early-stage non-small-cell lung cancer. J Clin Oncol. 2012;30:2731-8.

21. Zhong WZ. Chen KN, Chen C, et al Erlotinib versus gemcitabine plus cisplatin as neoadjuvant treatment of stage IIIA-N2 EGFR-mutant non-small-cell lung cancer (EMERGING-CTONG 1103): A randomized phase II study. J Clin Oncol. 2019;37:2235-45.

22. Mok TS. Wu YL, Ahn MJ, et al Osimertinib or platinum-pemetrexed in EGFR T790M-positive lung cancer. N Engl J Med. 2017;376: 629-40. 
23. Kim YI. Kang BC, Sung SH. Surgically resected gastric metastasis of pulmonary squamous cell carcinoma. World J Gastrointest Surg. 2013;5: 278-81.

24. Yang CJ. Hwang JJ, Kang WY, et al Gastro-intestinal metastasis of primary lung carcinoma: Clinical presentations and outcome. Lung Cancer. 2006;54: 319-23.

25. Ozdilekcan C. Songür N, Memiş L. Bozdogan N, Koksal AS. Ok U. Lung cancer associated with a single simultaneous solitary metastatic lesion in stomach: a case report with the review of literature. Tuberk Toraks. 2010;58:78-84.

26. McNeill PM. Wagman LD, Neifeld JP. Small bowel metastases from primary carcinoma of the lung. Cancer. 1987;59: 1486-9.

27. Antler AS. Ough Y, Pitchumoni CS. Davidian M, Thelmo W. Gastrointestinal metastases from malignant tumors of the lung. Cancer. 1982;49: 170-2.

28. He Y. Cui Y, Duan X. Primary lung squamous cell carcinoma with gastric metastasis: A case report. Thorac Cancer. 2019;10: 373-7.

29. Fu SW. Yang YH, Huang X. Clinicopathologic characteristics of gastric metastasis from primary lung cancer: a case report and review of the literature. World Chinese Journal of Digestology. 2014;22:2657-60.

30. Wang Y. An TT, Yang L. Primary lung cancer with gastrointestinal metastasis: 2 case report and literature review. Zhongguo Fei Ai Za Zhi. 2011;14:278 - 80.

31. Huang Q. Su XD, Bella AE, et al Clinicopathological features and outcome of gastric metastases from primary lung cancer: A case report and systematic review. Oncol Lett. 2015;9:1373-9.

32. Lee MH. Kim SR, Soh JS. A solitary gastric metastasis from pulmonary adenocarcinoma: a case report. Thorax. 2010;65:661-2.

33. Okazaki R. Ohtani $\mathrm{H}$, Takeda K, et al Gastric metastasis by primary lung adenocarcinoma. World J Gastrointest Oncol. 2010;2:395-8.

34. Altintas E. Sezgin O, Uyar B. Polat A. Acute upper gastrointestinal bleeding due to metastatic lung cancer an unusual case. Yonsei Med J. 2006;47:276-7.

35. Duan XQ. Zhao XH, Wang SH. An ALK-positive lung adenocarcinoma with gastric and skin metastasis: a case report and literature review. Ann Palliat Med. 2020.

36. Huang YM. Hsieh TY, Chen JR. Gastric and colonic metastases from primary lung adenocarcinoma: A case report and review of the literature. Oncol Lett. 2012;4:517 - 20.

37. Michael DR. Henry T. Not all gastric masses are gastric cancer. BMJ Case Rep. 2016;2016:bcr2015213535.

38. Kim YI. Kang BC, Sung SH. Surgically resected gastric metastasis of pulmonary squamous cell carcinoma. World J Gastrointest Surg. 2013;5:278 - 81.

39. Miyazaki J. Hirota S, Abe T. Metastasis of lung cancer to the gastrointestinal tract, presenting with a volcano-like ulcerated mass. Dig Endosc. 2015;27:397-8. 
40. Jujo T. Sakao S, Oide T. Tatsumi K. Metastatic gastric cancer from squamous cell lung carcinoma. Intern Med. 2012;51:1947-8.

41. Sharma P. Dwary AD, Khan EM. Serendipitous discovery of isolated gastric metastases from adenocarcinoma of the lung on staging 18F-FDG PET CT. Clin Nucl Med. 2017;42:807-8.

42. Bouzbib C. Chaput U, Jarrin I. Lavergne-slove A, Marteau P. Dray X. Bleeding from gastroduodenal metastases as the first manifestation of lung adenocarcinoma. Endoscopy. 2014;46:E474-5.

43. Katsenos S. Archondakis S. Solitary gastric metastasis from primary lung adenocarcinoma: a rare site of extra-thoracic metastatic disease. J Gastrointest Oncol. 2013;4:E11-5.

44. He Y,Cui Y, Duan XC. Liu CQ, Cai XQ. Primary lung squamous cell carcinoma with gastric metastasis: A case report. Thoracic Cancer. 2018.

45. Maeda J. Miyake M, Tokita K, et al Small cell lung cancer with extensive cutaneous and gastric metastases. Intern Med. 1992;31:1325-8.

46. Geoffrey RO. Hu YB, Mileham KF, et al Assessment of Resistance Mechanisms and Clinical Implications inPatients With EGFR T790M - Positive Lung Cancer and Acquired Resistance to Osimertinib. JAMA Oncology. 2018.

47. Offin M. Chan JM, Tenet M, et al Concurrent RB1 and TP53 alterations define a subset of EGFRmutant lung cancers at risk for histologic transformation and inferior clinical outcomes. J Thorac Oncol. (2019) 14:1784-93.

\section{Figures}
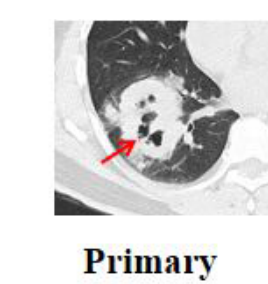

lesion

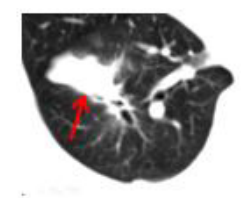

PR to gefitinib

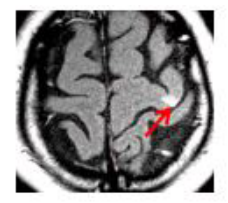

Postoperative recurrence

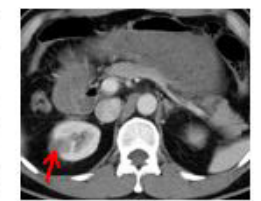

PD to chemotherapy

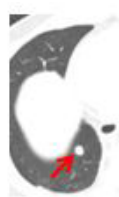

PD to osimertinib

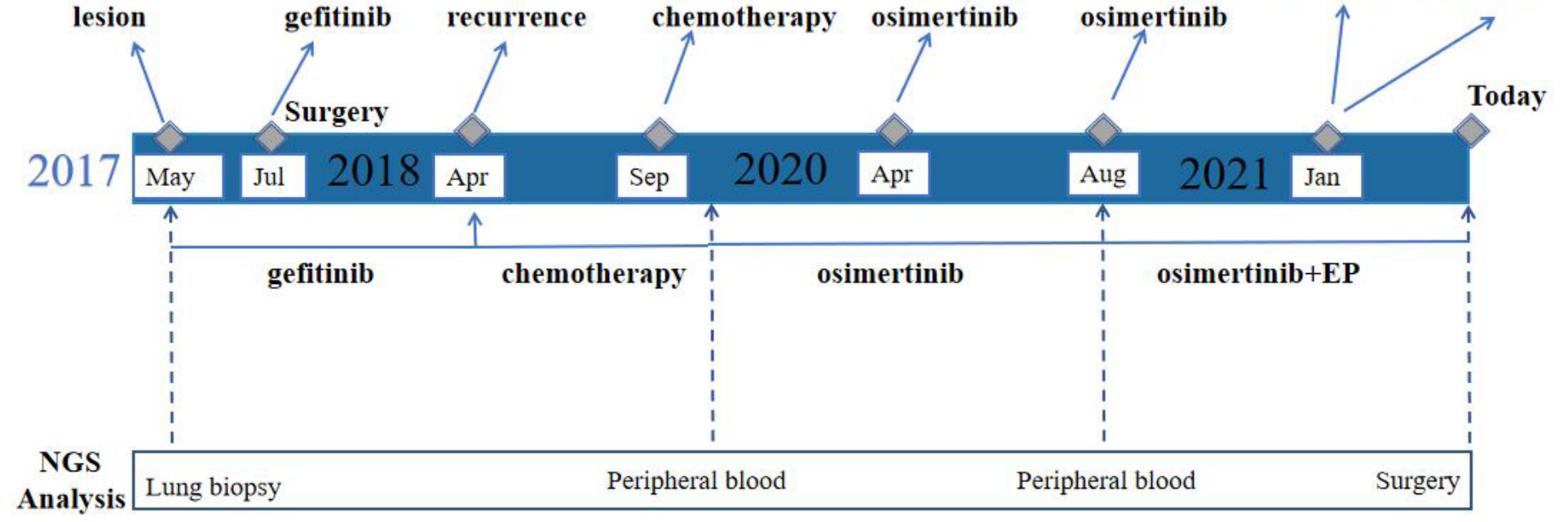

Figure 1 
Timeline of the clinical course of the patient. Arrows indicate the link between the clinical event and the date of NGS time point.
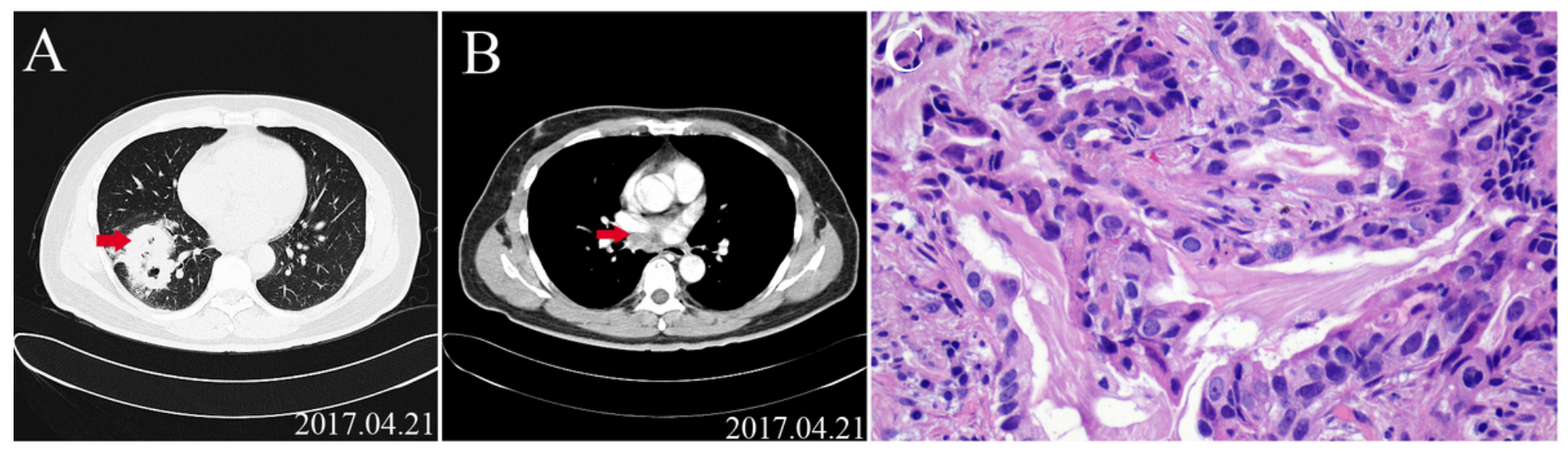

Figure 2

Computed tomography (CT) scan. a Chest scan indicating the presence of a nodule of $5.2 \times 5.1 \mathrm{~cm}$ in the right lower lobe of the lung. b Subcarinal lymph node metastasis. c Lung specimen showing cancer cell infiltration (hematoxylin and eosin staining, 40x magnification).
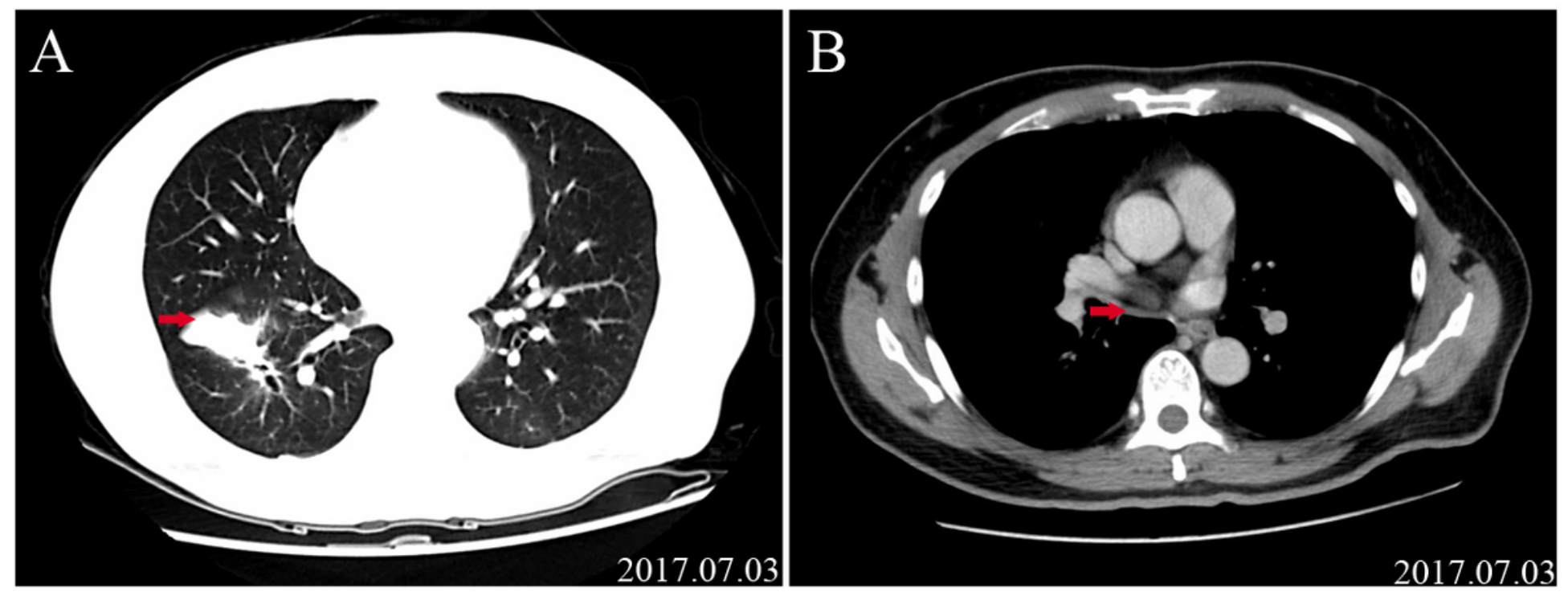

\section{Figure 3}

Computed tomography (CT) scan. a Chest scan indicating the presence of a nodule of $3.1 \times 2.4 \mathrm{~cm}$ in the right lower lobe indicating the decrease in its mass after the use of gefitinib as neoadjuvant treatment. $b$ Subcarinal lymph node metastasis disappearance after the use of gefitinib as neoadjuvant treatment. 

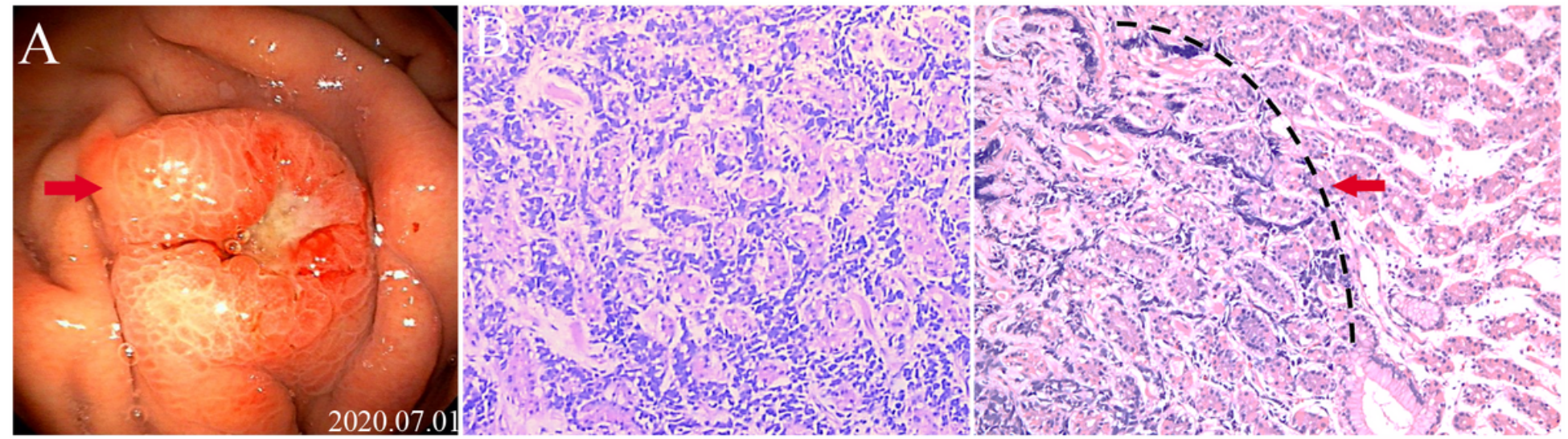

\section{Figure 4}

a Gastroscopy revealing a protruding lesion in the greater curvature of the stomach measuring $0.6 \times 0.4$ $\mathrm{cm}$, with mucosa erosion and hyperemia. b Gastric specimen showing cancer cell infiltration (hematoxylin and eosin staining, 40x magnification). c Gastric specimen showing clear boundaries between the cancer tissue and normal gastric gland (hematoxylin and eosin staining 40x magnification).
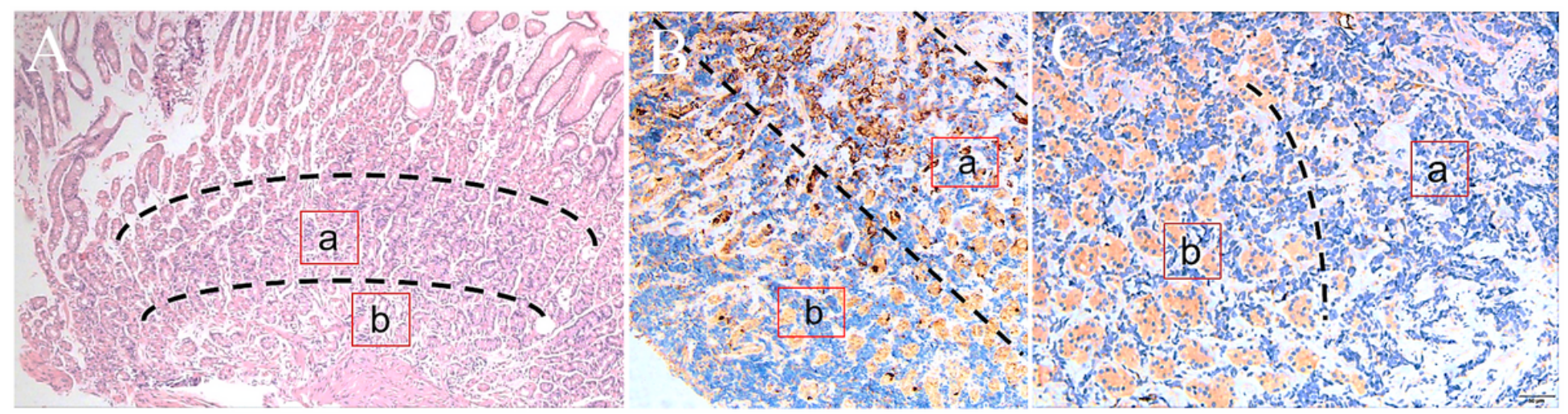

\section{Figure 5}

a Carcinoma cell infiltration into the submucosa of the stomach (hematoxylin and eosin staining, 40x magnification). b Representative image of CgA staining in tumor cells. c Representative image of CK7 staining in tumor cells. 200x magnification. a: near the mucosa. b: near the muscle. 

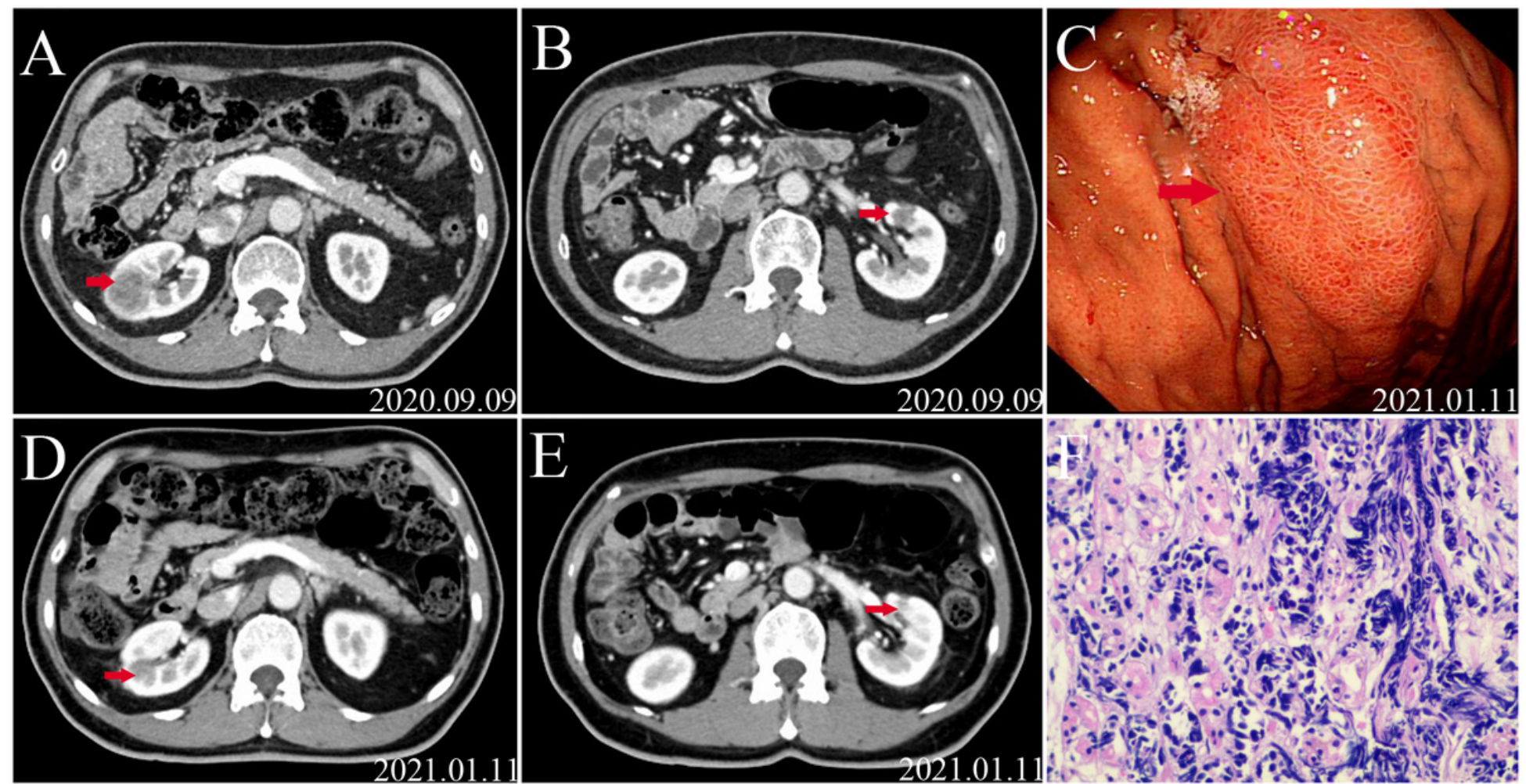

Figure 6

Computed tomography (CT) scan. a Abdominal scan showing the nodule in the right kidney before EP chemotherapy. b Abdominal scan showing the nodule in the left kidney before EP chemotherapy. $c$ Gastroscopy revealing the disappearance of the protruding lesion in the greater curvature of the stomach. $\mathrm{d}$ Abdominal scan showing the disappearance of the nodule in the right kidney after EP chemotherapy. e Abdominal scan showing the disappearance of the nodule in the left kidney after EP chemotherapy. $f$ Gastric specimen showing carcinoma cell infiltration after EP chemotherapy.

\section{Supplementary Files}

This is a list of supplementary files associated with this preprint. Click to download.

- Onlinetable1.png 